2. To: (Receiving Organization)

Distribution

5. Proj./Prog./Dept./Div.:

Tank 241-SX-106

8. Originator Remarks:

\section{Fn Appoval/Release}

11. Receiver Remarks:
3. From: (Originating organization)

M. J. Kupfer, LMHC, H5-49

6. Design Authority/ Design Agent/Cog. Engr.:

77. J. Kupper
4. Related EDT No.:

NA

7. Purchase Order No.:

NA

9. Equip./Component No.: NA

10. System/Bldg./Facility: NA

12. Major Assm. Dwg. No.:

NA

13. Permit/Permit Application No.:

$\mathrm{NA}$

14. Required Response Date:

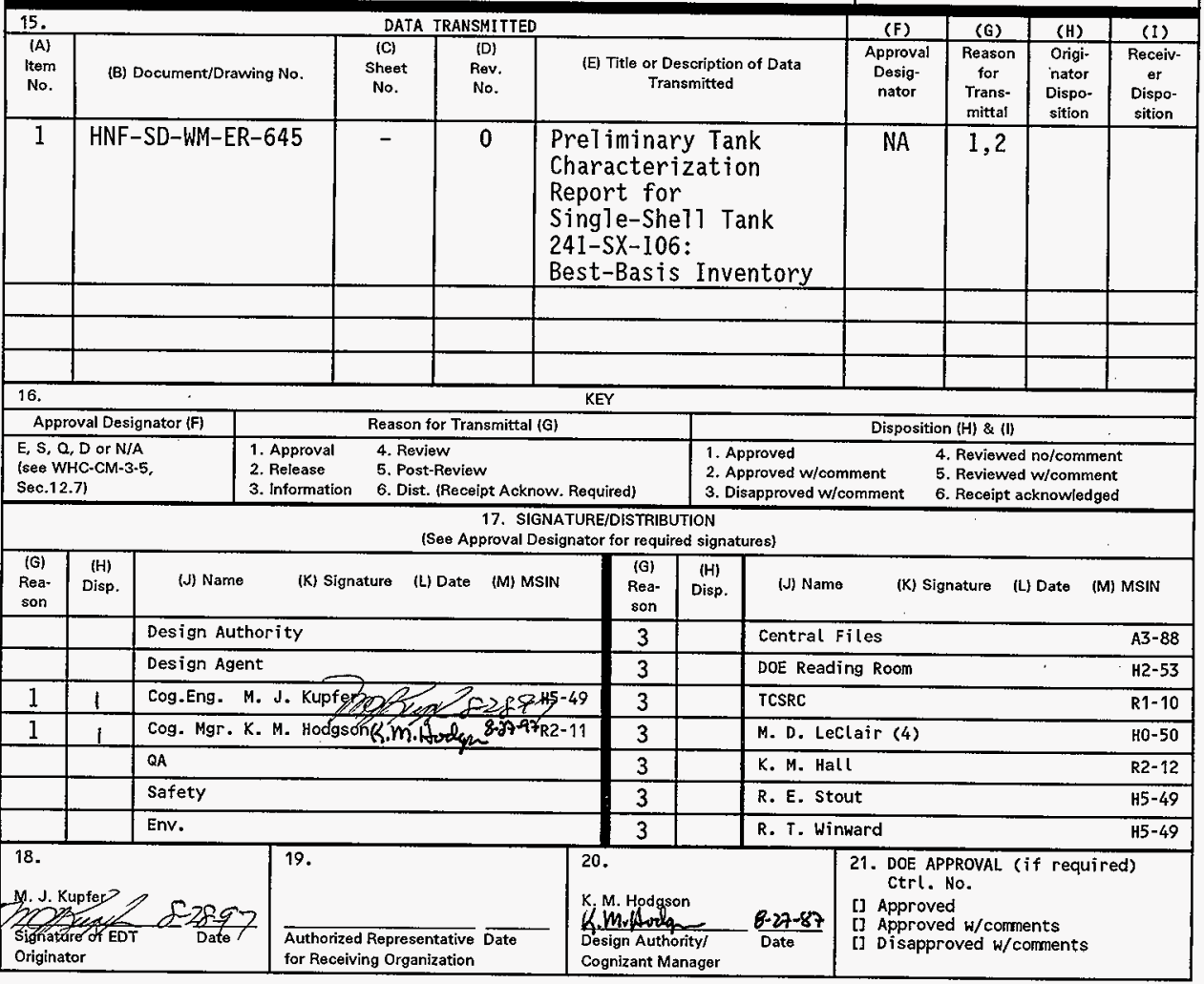




\title{
Preliminary Tank Characterization Report for Single-Shell Tank 241-SX-106: Best-Basis Inventory
}

\author{
R. E. Stout (Meier Associates), R. T. Winward (Meier Associates) and \\ M. J. Kupfer \\ Lockheed Martin Hanford Corporation, Richland, WA 99352 \\ U.S. Department of Energy Contract DE-AC06-96RL13200
}

EDT/ECN: 622677

Org Code: 74610

B\&R Code: EW3120074
UC: 712

Charge Code: $N 4 G 3 A$

Total Pages: 2527

Key Words: TCR, best-basis inventory

Abstract: An effort is underway to provide waste inventory estimates that will serve as standard characterization source terms for the varjous waste management activities. As part of this effort, an evaluation of available information for single-shel1 tank 241-SX-106 was performed, and a best-basis inventory was established. This work follows the methodology that was established by the standard inventory task.

TRADEMARK DISCLAIMER. Reference herein to any specific commercial product, process, or service by trade name, trademark, manufacturer, or otherwise, does not necessarily constitute or imply its endorsement, recommendation, or favoring by the United states Government or any agency thereof or its contractors or subcontractors.

Printed in the United States of America. To obtain copies of this document, contact: Document Control Services, P.0. Box 950, Mailstop H6-08, Richland WA 99352, Phone (509) 372-2420;

Fax (509) 376-4989.
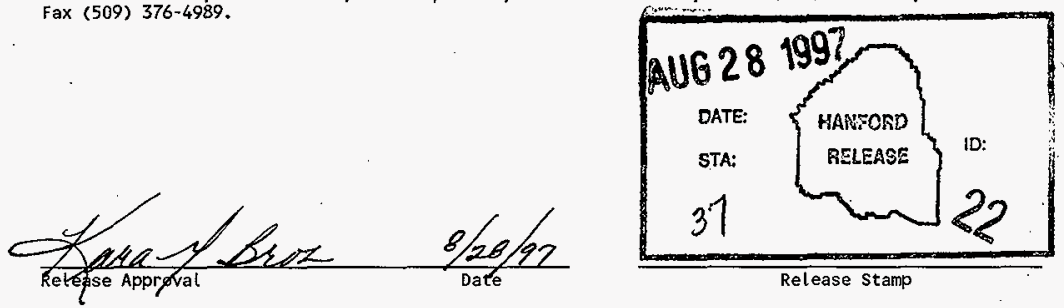

Release Stamp 


\section{PRELIMINARY TANK CHARACTERIZATION REPORT FOR SINGLE-SHELL TANK 241-SX-106: BEST-BASIS INVENTORY}

August 1997

M. J. Kupfer

Lockheed Martin Hanford Corporation

Richland, Washington

R. E. Stout

R. T. Winward

Meier Associates

Richland, Washington

Prepared for

U.S. Department of Energy

Richland, Washington 
HNF-SD-WM-ER-645

Revision 0

This page intentionally left blank. 
HNF-SD-WM-ER-645

Revision 0

\section{PRELIMINARY TANK CHARACTERIZATION REPORT \\ FOR SINGLE-SHELL TANK 241-SX-106: \\ BEST-BASIS INVENTORY}

This document is a preliminary Tank Characterization Report (TCR). It only contains the current best-basis inventory (Appendix D) for single-shell tank 241-SX-106. No TCRs have been previously issued for this tank, and current core sample analyses are not available. The best-basis inventory, therefore, is based on an engineering assessment of waste type, process flowsheet data, early sample data, and/or other available information.

The Standard Inventories of Chemicals and Radionuclides in Hanford Site Tank Wastes (Kupfer et al. 1997) describes standard methodology used to derive the tank-by-tank best-basis inventories. This preliminary TCR will be updated using this same methodology when additional data on tank contents become available.

\section{REFERENCE}

Kupfer, M. J., A. L. Boldt, B. A. Higley, K. M. Hodgson, L. W. Shelton, B. C. Simpson, and R. A. Watrous (LMHC), S. L. Lambert, and D. E. Place (SESC), R. M. Orme (NHC), G. L. Borsheim (Borsheim Associates), N. G. Colton (PNNL), M. D. LeClair (SAIC), R. T. Winward (Meier Associates), and W. W. Schulz (W'2 S Corporation), 1997, Standard Inventories of Chemicals and Radionuclides in Hanford Site Tank Wastes, HNF-SD-WM-TI-740, Rev. 0, Lockheed Martin Hanford Corporation, Richland, Washington. 
HNF-SD-WM-ER-645

Revision 0

This page intentionally left blank. 
HNF-SD-WM-ER-645

Revision 0

APPENDIX D

\section{EVALUATION TO ESTABLISH BEST-BASIS INVENTORY FOR SINGLE-SHELL TANK 241-SX-106}


HNF-SD-WM-ER-645

Revision 0

This page intentionally left blank. 
HNF-SD-WM-ER-645

Revision 0

APPENDIX D

\section{EVALUATION TO ESTABLISH BEST-BASIS INVENTORY FOR SINGLE-SHELL TANK 241-SX-106}

An effort is underway to provide waste inventory estimates that will serve as standard characterization source terms for the various waste management activities (Hodgson and LeClair 1996). As part of this effort, an evaluation of available information for single-shell tank 241-SX-106 was performed, and a best-basis inventory was established. This work, detailed in the following sections, follows the methodology that was established by the standard inventory task.

\section{D1.0 CHEMICAL INFORMATION SOURCES}

There is no previous Tank Characterization Report (TCR) for tank 241-SX-106. Available information for tank 241-SX-106 includes the following:

- Tank Characterization Reports from S and U Tank Farm tanks with similar Supernatant Mixing Model S Plant (SMMS) salt cake waste and first-cycle Reduction and Oxidation (REDOX) Plant (R1) sludge waste types.

- The Hanford Defined Waste (HDW) model document (Agnew et al. 1996) provides tank content estimates, derived from the HDW model, in terms of component concentrations and inventories.

\section{D2,0 COMPARISON OF COMPONENT INVENTORY VALUES}

The inventories generated by the HDW model, are shown in Tables D2-1 and D2-2. No samples have been taken from tank 241-SX-106 that can be used to estimate a tank inventory for comparison with the HDW model estimate. The tank volume used to generate the HDW inventory is $2,037 \mathrm{~kL}$ (538 kgal) total waste which partitions into $3.78 \mathrm{~kL}$ (1 kgal) sludge, 2,032 kL (537 kgal) salt cake (Agnew et al. 1996), and differs slightly from the $2,037 \mathrm{~kL}$ (538 kgal) of total waste of which there is $45 \mathrm{~kL}$ (12 kgal) sludge, $1,760 \mathrm{~kL}$ (465 kgal) salt cake and $231 \mathrm{~kL}$ (61 kgal) supernatant reported by Hanlon (1996). The HDW sludge waste density used is $1.49 \mathrm{~g} / \mathrm{mL}$ and the salt cake density used is $1.53 \mathrm{~g} / \mathrm{mL}$ (The chemical species are reported without charge designation per the best-basis inventory convention.) 


\section{Revision 0}

Table D2-1. Hanford Defined Waste-Based Inventory Estimates for Nonradioactive Components in Tank 241-SX-106.

\begin{tabular}{|c|c|c|c|}
\hline Analyte & $\begin{array}{l}\mathrm{HDW}^{\mathrm{a}} \text { inventory } \\
\text { estimate }(\mathrm{kg})\end{array}$ & Analyte & $\begin{array}{l}\text { HDW }^{\mathrm{a}} \text { inventory } \\
\text { estimate }(\mathrm{kg})\end{array}$ \\
\hline $\mathrm{Al}$ & 83,600 & $\mathrm{NO}_{3}$ & 648,000 \\
\hline $\mathrm{Bi}$ & 578 & $\mathrm{OH}$ & 228,000 \\
\hline $\mathrm{Ca}$ & 2,750 & oxalate & 6.86 \\
\hline $\mathrm{Cl}$ & 14,500 & $\mathrm{~Pb}$ & 403 \\
\hline $\mathrm{Cr}$ & 6,030 & $\mathrm{P}$ as $\mathrm{PO}_{4}$ & 17,600 \\
\hline $\mathrm{F}$ & 2,960 & $\mathrm{Si}$ & 4,420 \\
\hline $\mathrm{Fe}$ & 844 & $\mathrm{~S}$ as $\mathrm{SO}_{4}$ & 46,600 \\
\hline $\mathrm{Hg}$ & 3.64 & $\mathrm{Sr}$ & 2.66 \\
\hline $\mathrm{K}$ & 4,340 & TIC as $\mathrm{CO}_{3}$ & 52,800 \\
\hline $\mathrm{La}$ & 12.7 & TOC & 25,300 \\
\hline $\mathrm{Mn}$ & 430 & $\mathrm{U}_{\text {TOTAL }}$ & 5,870 \\
\hline $\mathrm{Na}$ & 532,000 & $\mathrm{Zr}$ & 174 \\
\hline $\mathrm{Ni}$ & 761 & $\mathrm{H}_{2} \mathrm{O}(\mathrm{Wt} \%)$ & 38.2 \\
\hline $\mathrm{NO}_{2}$ & 212,000 & density $(\mathrm{kg} / \mathrm{L})$ & 1.53 \\
\hline
\end{tabular}

HDW $=$ Hanford Defined Waste

${ }^{2}$ Agnew et al. (1996)

Table D2-2. Predicted Inventory Estimates for Radioactive Components in Tank 241-SX-106.

\begin{tabular}{|c|c||c|c|}
\hline Analyte & $\begin{array}{c}\mathrm{HDW}^{\text {a }} \text { inventory } \\
\text { estimate }(\mathrm{Ci})\end{array}$ & Analyte & $\begin{array}{c}\text { HDW inventory } \\
\text { estimate }(\mathrm{Ci})\end{array}$ \\
\hline${ }^{90} \mathrm{Sr}$ & 239,000 & ${ }^{239 / 240} \mathrm{Pu}$ & 164 \\
\hline${ }^{137} \mathrm{Cs}$ & 499,000 & & \\
\hline
\end{tabular}

HDW $=$ Hanford Defined Waste

Agnew et al. (1996), radionuclides decayed to January 1, 1994. 
HNF-SD-WM-ER-645

Revision 0

\section{D3.0 COMPONENT INVENTORY EVALUATION}

\section{D3.1 WASTE HISTORY TANK 241-SX-106}

Tank 241-SX-106 was filled with waste from the REDOX Plant from the third quarter of 1954 until the fourth quarter of 1971 . From 1963 until 1971 there were waste transfers primarily involving recycled supernatant evaporator bottoms $(\mathrm{EB})$ in and out of tank 241-SX-106 with 12 other SX Tank Farm tanks. The tank was removed from service and labeled inactive in 1980 and was partially isolated in 1985 . The tank level was adjusted in December 1979 and October 1980.

\section{D3.2 CONTRIBUTING WASTE TYPES}

Agnew et al. (1996): RSltCk, SMMS1, SMMS2

Hill et al. (1995): R, EB, RIX

The HDW model (Agnew et al. 1996) predicts that the tank contains a total of $2,037 \mathrm{~kL}$ ( $538 \mathrm{kgal})$ of waste which consists of RSltCk waste $(3.78 \mathrm{~kL}$ [1 kgal], R1), and $2,032 \mathrm{~kL}(537 \mathrm{kgal})$ of salt cake predicted from the SMM. Although the total waste volume for SMM salt cake listed in Appendix E of Hanlon (1996) is $2,032 \mathrm{~kL}$ (537 kgal), the tank layer model (TLM) in Appendix C of Hanlon only accounts for $1,820 \mathrm{~kL}$ (476 kgal) of SMM salt cake. The Appendix E of Hanlon (1997) value is assumed correct.

The Sort on Radioactive Waste Type (SORWT) model (Hill et al. 1995) lists .R (high-level REDOX process waste), and Evaporator Bottoms (EB) as the primary and secondary waste types respectively. EB waste is the SORWT definition for salt cake that is equivalent to the SMM waste type. Hill et al. also lists REDOX process ion exchange (RIX) as a tertiary waste contributor.

Hanlon (1996) indicates $2,037 \mathrm{~kL}$ (538 kgal) of waste which consists of, $45 \mathrm{~kL}$ (12 kgal) of sludge, $1,760 \mathrm{~kL}$ (465 kgal) of salt cake, and $231 \mathrm{~kL}$ (61 kgal) of supernatant. No description of the source of the sludge and salt cake are given.

\subsection{INVENTORY EVALUATION}

The following evaluation provides an engineering assessment of tank 241-SX-106 contents. For this evaluation, the following assumptions and observations are made:

- Tank volume used is the one listed in both Hanlon (1996) and Agnew et al. (1996), 2,037 kL (538 kgal). 
HNF-SD-WM-ER-645

Revision 0

- Only the SMMS1 and SMMS2 and R waste streams contributed to solids formation.

- The $231 \mathrm{~kL}$ (61 kgal) supernatant listed by Hanlon (1996) is assumed to be same composition as SMMS2.

- The Hanlon (1996) sludge volume (45 kL [12 kgal]) was assumed to be first-cycle REDOX process waste (R1) which cascaded into tank 241-SX-106 from tank 241-SX-105.

- The relative volumes of 1,760 kL (465 kgal) SMMS1 and SMMS2 salt cakes were determined from Appendix C of Agnew et al. (1996).

\section{D3.4 BASIS FOR CALCULATIONS USED IN THIS ENGINEERING EVALUATION}

Table D3-1 shows the engineering evaluation approaches used on tank 241-SX-106.

Table D3-1. Engineering Evaluation Approaches Used On 241-SX-106. (2 Sheets)

\begin{tabular}{|l|l|l|}
\hline \multicolumn{1}{|c|}{ Type of waste } & \multicolumn{1}{c|}{ How calculated } & \multicolumn{1}{c|}{ Check method } \\
\hline $\begin{array}{l}\text { Supernatant } \\
\text { Volume }=231 \mathrm{~kL} \\
(61 \mathrm{kgal})\end{array}$ & Not calculated. & $\begin{array}{l}\text { None, no sampling data } \\
\text { available. }\end{array}$ \\
\hline $\begin{array}{l}\text { Salt cake } \\
\text { Volume }=1,760 \mathrm{~kL} \\
(465 \mathrm{kgal})\end{array}$ & $\begin{array}{l}\text { Used sample-based concentrations } \\
\text { from tanks with SMMS1 and } \\
\text { SMMS2 salt cake waste. }\end{array}$ & $\begin{array}{l}\text { None, no sampling data } \\
\text { are available for this tank. }\end{array}$ \\
$\begin{array}{l}\text { Density }=1.63 \mathrm{~g} / \mathrm{mL} \\
\text { for SMMS1 waste and } \\
1.56 \mathrm{~g} / \mathrm{mL} \text { for SMMS2 } \\
\text { waste. }\end{array}$ & $\begin{array}{l}\text { SMMS1 318 kL (84 kgal) } \\
\text { SMMS2 1,673 kL (442 kgal) }\end{array}$ & \\
\hline $\begin{array}{l}\text { Sludge } \\
\text { Volume }=45 \mathrm{~kL} \\
(12 \mathrm{kgal})\end{array}$ & $\begin{array}{l}\text { Used the average analyte } \\
\text { concentration from tanks } \\
241-S-102,241-S-104, \text { and } \\
\text { 241-S-107. All have sample data } \\
\text { and R1 waste. Only the segments } \\
\text { that are believed to have R1 waste } \\
\text { were used to calculate the } \\
\text { concentration from each tank. }\end{array}$ & $\begin{array}{l}\text { None, no sample-based } \\
\text { information is available } \\
\text { for this tank. }\end{array}$ \\
\hline
\end{tabular}

$\mathrm{R}=$ Reduction and Oxidation (REDOX) process sludge waste

SMMS = Supernatant Mixing Model S Plant. 


\section{D3.4.1 Basis for Salt Cake Calculations Used in this Engineering Evaluation.}

For this evaluation the methodology developed for SMMS1 and SMMS2 salt cake was used. This is based on comparing concentrations from $\mathrm{S}$ and $\mathrm{U}$ Tank Farm sample data shown in Table D3-2 and D3-3. Tanks 241-S-101 (Kruger et al. 1996), 241-S-102 (Eggers et al. 1996), 241-U-106 (Brown et al. 1997), and 241-U-109 (Baldwin and Stephens 1996) were used to produce the average salt cake analyte concentrations for SMMS1 salt cake and tanks 241-S-101 (Kruger et al. 1996), 241-S-102 (Eggers et al. 1996), 241-U-102 ( $\mathrm{Hu}$ et al. 1997), 241-U-107 (Jo et al. 1996), and 241-U-109 (Baldwin and Stephens 1996) for SMMS2 salt cake were used in this comparison. To calculate the average SMMS1 and SMMS2 concentration the waste volumes and predicted location from Agnew et al. for both the SMMS1 and SMMS2 layers in each tank was determined. The sample data were reviewed and using the segments that were located within the predicted location from Agnew et al. (1996), an average concentration was calculated. The concentrations from each tank and the segments used in the calculations are shown in Tables D3-2 and D3-3. For comparison the SMM salt cake composition predicted by the HDW model for tank 241-SX-106 is also shown.

Table D3-2. SMMS1 Salt Cake Concentrations. (2 Sheets)

\begin{tabular}{|c|c|c|c|c|c|c|}
\hline Analyte & $\begin{array}{c}241-\mathrm{S}-101 \\
\text { segments } \\
2 \mathrm{~L}-4 \mathrm{U}^{\mathrm{a}} \\
(\mu \mathrm{g} / \mathrm{g})\end{array}$ & $\begin{array}{c}241-\mathrm{S}-102 \\
\text { segments } \\
7 \mathrm{~L}-10 \mathrm{U}^{\mathrm{b}} \\
(\mu \mathrm{g} / \mathrm{g})\end{array}$ & $\begin{array}{c}241-\mathrm{U}-106 \\
\text { segments } \\
2 \mathrm{U}^{\circ}-4 \mathrm{~L}^{\circ} \\
(\mu \mathrm{g} / \mathrm{g})\end{array}$ & $\begin{array}{c}241-\mathrm{U}-109 \\
\text { segments } \\
5 \mathrm{U}_{-}-8 \mathrm{~L}^{\mathrm{d}} \\
(\mu \mathrm{g} / \mathrm{g})\end{array}$ & $\begin{array}{c}\text { Average } \\
\text { concentration } \\
(\mu \mathrm{g} / \mathrm{g})\end{array}$ & $\begin{array}{c}\text { HDW model SMM } \\
\text { concentration for } \\
\text { tank 241-SX-106 } \\
(\mu \mathrm{g} / \mathrm{g})\end{array}$ \\
\hline $\mathrm{Al}$ & 18,000 & 15,085 & 13,620 & 13,625 & 15,100 & 26,800 \\
\hline $\mathrm{Ag}$ & 12 & 17 & 16 & $\mathrm{NR}$ & 15 & $\mathrm{NR}$ \\
\hline $\mathrm{B}$ & 110 & 75 & 80 & $\mathrm{NR}$ & 88 & $\mathrm{NR}$ \\
\hline $\mathrm{Bi}$ & 71 & 76 & $<\mathrm{DL}$ & $<\mathrm{DL}$ & 73.5 & 186 \\
\hline $\mathrm{Ca}$ & 273 & 237 & 336 & $<\mathrm{DL}$ & 282 & 881 \\
\hline $\mathrm{Cl}$ & 4,500 & 4,099 & 2,926 & $\mathrm{NR}$ & 3,842 & 4,660 \\
\hline $\mathrm{Cr}$ & 10,000 & 4,359 & 3,170 & 4,233 & 5,440 & 1,920 \\
\hline $\mathrm{F}$ & 500 & 13,596 & 4,669 & $\mathrm{NR}$ & 6,255 & 950 \\
\hline $\mathrm{Fe}$ & 508 & 1,298 & 3,096 & $<\mathrm{DL}$ & 1,630 & 270 \\
\hline $\mathrm{K}$ & 1,109 & 898 & 1,309 & $\mathrm{NR}$ & 1,110 & 1,390 \\
\hline $\mathrm{La}$ & $<\mathrm{DL}$ & 37 & 43 & $\mathrm{NR}$ & 40 & 4.06 \\
\hline $\mathrm{Mn}$ & 266 & 597 & 1,189 & $<\mathrm{DL}$ & 684 & 138 \\
\hline $\mathrm{Na}$ & 150,000 & 189,500 & 170,500 & 218,300 & 182,000 & 171,000 \\
\hline $\mathrm{Ni}$ & 114 & 49 & 304 & $<\mathrm{DL}$ & 155 & 243 \\
\hline $\mathrm{NO}_{2}$ & 91,000 & 40,100 & 56,000 & 42,900 & 57,500 & 68,100 \\
\hline $\mathrm{NO}$ & 110,000 & 99,200 & 147,200 & 297,000 & 163,000 & 208,000 \\
\hline $\mathrm{Pb}$ & 91 & 137 & 348 & $\mathrm{NR}$ & 192 & 129 \\
\hline $\mathrm{PO}$ & 9,500 & 114,500 & 5,888 & 5,970 & 34,000 & 5,640 \\
\hline
\end{tabular}


HNF-SD-WM-ER-645

Revision 0

Table D3-2. SMMS1 Salt Cake Concentrations. (2 Sheets)

\begin{tabular}{|c|c|c|c|c|c|c|}
\hline Analyte & $\begin{array}{c}241-\mathrm{S}-101 \\
\text { segments } \\
2 \mathrm{~L}-4 \mathrm{U}^{\mathrm{a}} \\
(\mu \mathrm{g} / \mathrm{g})\end{array}$ & $\begin{array}{c}241-S-102 \\
\text { segments } \\
7 \mathrm{~L}-10 \mathrm{U}^{\mathrm{b}} \\
(\mu \mathrm{g} / \mathrm{g})\end{array}$ & $\begin{array}{c}241-\mathrm{U}-106 \\
\text { segments } \\
2 \mathrm{U}-4 \mathrm{~L}^{\mathrm{c}} \\
(\mu \mathrm{g} / \mathrm{g})\end{array}$ & $\begin{array}{c}241-\mathrm{U}-109 \\
\text { segments } \\
5 \mathrm{U}-8 \mathrm{~L}^{\mathrm{d}} \\
(\mu \mathrm{g} / \mathrm{g})\end{array}$ & $\begin{array}{c}\text { Average } \\
\text { concentration } \\
(\mu \mathrm{g} / \mathrm{g})\end{array}$ & $\begin{array}{c}\text { HDW model SMM } \\
\text { concentration for } \\
\text { tank } 241-\mathrm{SX}-106^{\mathrm{f}} \\
(\mu \mathrm{g} / \mathrm{g})\end{array}$ \\
\hline $\mathrm{P}$ & 2,290 & 33,900 & 1,949 & $<\mathrm{DL}$ & 12,700 & NR \\
\hline$S$ & 5,940 & 2,683 & 3,878 & NR & 4,170 & NR \\
\hline $\mathrm{Si}$ & 5,269 & 517 & 176 & $<\mathrm{DL}$ & 1,990 & 1,420 \\
\hline $\mathrm{SO}_{4}$ & 20,700 & 12,500 & 10,774 & 11,100 & 13,800 & 15,000 \\
\hline $\mathrm{Sr}$ & 7 & $<\mathrm{DL}$ & $<\mathrm{DL}$ & NR & 7 & 0.855 \\
\hline TOC & 1,900 & 5,340 & 24,626 & 3,920 & 8,950 & 8,130 \\
\hline $\bar{U}$ & 560 & 1,403 & 781 & $<\mathrm{DL}$ & 914 & 1,880 \\
\hline $\mathrm{Zn}$ & 30 & 32 & 54 & $<\mathrm{DL}$ & 39 & NR \\
\hline $\mathrm{Zr}$ & 14 & 39 & 88 & NR & 47 & 55.9 \\
\hline Oxalate & 15,400 & 15,700 & 9,880 & NR & 13,700 & 2.20 \\
\hline $\begin{array}{c}\text { Density } \\
\mathrm{g} / \mathrm{mL}\end{array}$ & 1.58 & 1.69 & 1.57 & 1.67 & 1.63 & 1.46 \\
\hline \multicolumn{7}{|c|}{ Radionuclides $^{\mathrm{g}}(\mu \mathrm{Ci} / \mathrm{g})$} \\
\hline${ }^{90} \mathrm{Sr}$ & 252 & 23 & 77 & 9 & 90 & 76.7 \\
\hline${ }^{137} \mathrm{Cs}$ & 175 & 121 & 175 & 142 & 153 & 160 \\
\hline
\end{tabular}

$\angle \mathrm{DL}=$ Less then the Detectable Limit.

HDW $=$ Hanford Defined Waste

$\mathrm{NR}=$ Not reported

SMMS1 = Supernatant Mixing Model 242-S Evaporator salt cake generated from 1973 until 1976

${ }^{a}$ Kruger et al. (1996)

${ }^{b}$ Eggers et al. (1996)

${ }^{\circ}$ Brown et al. (1997)

dBaldwin and Stephens (1996)

'Average of tank 241-S-101, 241-S-102, 241-U-106, and 241-U-109 concentrations

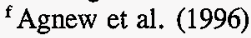

${ }^{\mathrm{g}}$ Radionuclides are reported as of the date of sample analysis. 
HNF-SD-WM-ER-645

Revision 0

Table D3-3. SMMS2 Salt Cake Concentrations. (2 Sheets)

\begin{tabular}{|c|c|c|c|c|c|c|c|}
\hline Analyte & $\begin{array}{c}241-\mathrm{S}-101 \\
\text { segments } \\
1 \mathrm{U}-2 \mathrm{U}^{\mathrm{a}} \\
(\mu \mathrm{g} / \mathrm{g})\end{array}$ & $\begin{array}{c}241-\mathrm{S}-102 \\
\text { segments } \\
2 \mathrm{U}-5 \mathrm{~L}^{\mathrm{b}} \\
(\mu \mathrm{g} / \mathrm{g})\end{array}$ & $\begin{array}{c}241-\mathrm{U}-102 \\
\text { segments } \\
2 \mathrm{U}^{\mathrm{c}} \\
(\mu \mathrm{g} / \mathrm{g})\end{array}$ & $\begin{array}{c}241-\mathrm{U}-107 \\
\text { segments } \\
2 \mathrm{U}-6 \mathrm{~L}^{\mathrm{d}} \\
(\mu \mathrm{g} / \mathrm{g})\end{array}$ & $\begin{array}{c}241-\mathrm{U}-109 \\
\text { segments } \\
1 \mathrm{~L}-4 \mathrm{U}^{\mathrm{e}} \\
(\mu \mathrm{g} / \mathrm{g})\end{array}$ & $\begin{array}{c}\text { HDW model } \\
\text { SSMS2 } \\
\text { Salt Cake } \\
\text { Average } \\
(\mu \mathrm{g} / \mathrm{g})\end{array}$ & $\begin{array}{c}\text { concentration } \\
\text { for tank } \\
241-\mathrm{SX}-106 \\
(\mu \mathrm{g} / \mathrm{g})\end{array}$ \\
\hline $\mathrm{Al}$ & 16,925 & 7,450 & 10,505 & 10,612 & 9,487 & 10,996 & 26,800 \\
\hline $\mathrm{Ag}$ & 12 & 17 & 13 & 16 & $\mathrm{NR}$ & 14 & $\mathrm{NR}$ \\
\hline $\mathrm{B}$ & 111 & 58 & 67 & 89 & $\mathrm{NR}$ & 81 & $\mathrm{NR}$ \\
\hline $\mathrm{Bi}$ & 51 & $<\mathrm{DL}$ & $<\mathrm{DL}$ & 270 & $<\mathrm{DL}$ & 161 & 186 \\
\hline $\mathrm{Ca}$ & 274 & 233 & 310 & 298 & $<\mathrm{DL}$ & 279 & 881 \\
\hline $\mathrm{Cl}$ & 4,607 & 2,981 & 4,550 & 2,515 & 3,560 & 3,643 & 4,660 \\
\hline $\mathrm{Cr}$ & 8,163 & 1,577 & 2,417 & 2,570 & 2,570 & 3,459 & 1,920 \\
\hline $\mathrm{F}$ & 638 & 267 & 896 & 501 & 299 & 520 & 950 \\
\hline $\mathrm{Fe}$ & 453 & 65 & 565 & 767 & 1,630 & 696 & 270 \\
\hline $\mathrm{K}$ & 1,225 & 748 & 1,360 & 914 & $\mathrm{NR}$ & 1,062 & 1,390 \\
\hline $\mathrm{Mn}$ & 541 & 26 & 137 & 330 & $<\mathrm{DL}$ & 258 & 138 \\
\hline $\mathrm{Na}$ & 153,000 & 207,000 & 176,000 & 205,667 & 237,333 & 195,800 & 171,000 \\
\hline $\mathrm{Ni}$ & 115 & 19 & 77 & 56 & $<\mathrm{DL}$ & 67 & 243 \\
\hline $\mathrm{NO}_{2}$ & 58,150 & 28,939 & 36,250 & 27,600 & 42,900 & 38,768 & 68,100 \\
\hline $\mathrm{NO}_{3}$ & 218,500 & 514,000 & 293,000 & 455,333 & 407,333 & 377,633 & 208,000 \\
\hline $\mathrm{Pb}$ & 66 & 47 & $<\mathrm{DL}$ & 149 & $\mathrm{NR}$ & 87 & 129 \\
\hline $\mathrm{PO}_{4}$ & 9,230 & 15,589 & 19,950 & 13,509 & 5,970 & 12,850 & 5,640 \\
\hline $\mathrm{P}$ & 2,333 & 2,860 & 6,187 & 2,580 & 7,780 & 4,348 & $\mathrm{NR}$ \\
\hline $\mathrm{S}$ & 4,713 & 1,325 & 4,037 & 1,090 & $\mathrm{NR}$ & 2,791 & $\mathrm{NR}$ \\
\hline $\mathrm{Si}$ & $<\mathrm{DL}$ & 219 & 148 & 194 & 1,220 & 445 & 1,420 \\
\hline $\mathrm{SO} \mathrm{F}_{4}$ & 21,185 & 8,553 & 12,785 & 4,112 & 11,000 & 11,527 & 15,000 \\
\hline $\mathrm{Sr}$ & 48 & $<\mathrm{DL}$ & $<\mathrm{DL}$ & 9 & $\mathrm{NR}$ & 28 & 0.855 \\
\hline $\mathrm{TOC}$ & $\mathrm{NR}$ & 1,898 & 6,417 & 2,414 & 2,330 & 3,265 & 8,130 \\
\hline $\mathrm{U}$ & 1,497 & $<\mathrm{DL}$ & $<\mathrm{DL}$ & 430 & $<\mathrm{DL}$ & 964 & 1,880 \\
\hline $\mathrm{Zn}$ & 33 & 21 & 33 & 29 & $\mathrm{NR}$ & 29 & $\mathrm{NR}$ \\
\hline
\end{tabular}


HNF-SD-WM-ER-645

Revision 0

Table D3-3. SMMS2 Salt Cake Concentrations. (2 Sheets)

\begin{tabular}{|c|c|c|c|c|c|c|c|}
\hline Analyte & $\begin{array}{c}241-\mathrm{S}-101 \\
\text { segments } \\
1 \mathrm{U}-2 \mathrm{U}^{2} \\
(\mu \mathrm{g} / \mathrm{g})\end{array}$ & $\begin{array}{c}241-\mathrm{S}-102 \\
\text { segments } \\
2 \mathrm{U}-5 \mathrm{~L}^{\mathrm{b}} \\
(\mu \mathrm{g} / \mathrm{g})\end{array}$ & $\begin{array}{c}241-\mathrm{U}-102 \\
\text { segments } \\
2 \mathrm{U}^{\mathrm{c}} \\
(\mu \mathrm{g} / \mathrm{g})\end{array}$ & $\begin{array}{c}241-\mathrm{U}-107 \\
\text { segments } \\
2 \mathrm{U}-\mathrm{L}^{\mathrm{d}} \\
(\mu \mathrm{g} / \mathrm{g})\end{array}$ & $\begin{array}{c}241-\mathrm{U}-109 \\
\text { segments } \\
1 \mathrm{~L}-4 \mathrm{U}^{\mathrm{e}} \\
(\mu \mathrm{g} / \mathrm{g})\end{array}$ & $\begin{array}{c}\text { Average } \\
\text { concentration } \\
(\mu \mathrm{g} / \mathrm{g})\end{array}$ & $\begin{array}{c}\text { HDW model } \\
\text { SSMS2 } \\
\text { Salt Cake } \\
\text { concentration } \\
\text { for tank } \\
241-\mathrm{SX}-106 \\
(\mu \mathrm{g} / \mathrm{g})\end{array}$ \\
\hline $\mathrm{Zr}$ & 13 & $<\mathrm{DL}$ & <DL & 13 & $\mathrm{NR}$ & 13 & 55.9 \\
\hline \multicolumn{7}{|l|}{ Radionuclide } \\
\hline${ }^{90} \mathrm{Sr}$ & 252 & $\mathrm{NR}$ & $<\mathrm{DL}$ & 0.297 & 4.81 & 86 & \\
\hline${ }^{137} \mathrm{Cs}$ & 160.15 & $\mathrm{NR}$ & 136.5 & 62.06 & 89.1 & 112 & \\
\hline
\end{tabular}

$\angle \mathrm{DL}=$ Less than detectable limit

HDW $=$ Hanford Defined Waste

NR $=$ Not reported

SMMS2 = Supernatant Mixing Model 242-S Evaporator salt cake generated

from 1977 until 1980

a Kruger et al. (1996)

${ }^{b}$ Eggers et al. (1996)

${ }^{c} \mathrm{Hu}$ et.al. (1997)

Jo et al. (1996)

'Baldwin and Stephens (1996)

${ }^{\mathrm{f}}$ Average of tank 241-S-101, 241-S-102, 241-U-102, 241-U-107, and 241-U-109

concentrations

${ }^{8}$ Radionuclides are reported as of the date of sample analysis.

\section{D3.4.2 Basis for Sludge Calculations Used in This Engineering Evaluation.}

Data from tanks 241-S-102 (Eggers et al. 1996), 241-S-104 (DiCenso et al. 1994), and 241-S-107 (Simpson et al. 1996) were used to produce average analyte concentrations for R1 sludge waste. To calculate the average concentration, the volumes and predicted location of the sludge were taken from Agnew et al. (1996) for the tanks with R1 waste. The sample data were then reviewed, and only the segments that were located within the predicted sludge location from Agnew et al. were used in deriving an average concentration. The average concentration from each tank and the segments used in the calculation are shown below in Table D3-4. For comparison the average sludge layer composition predicted by the HDW model for tank 241-SX-106 is also shown. 
HNF-SD-WM-ER-645

Revision 0

Table D3-4. R1 Sludge Concentration Estimate. (2 Sheets)

\begin{tabular}{|c|c|c|c|c|c|}
\hline Analyte & $\begin{array}{c}241-\mathrm{S}-101 \\
\text { segments } \\
7 \mathrm{U}-8 \mathrm{~L}^{\mathrm{a}} \\
(\mu \mathrm{g} / \mathrm{g})\end{array}$ & $\begin{array}{c}241-\mathrm{S}-104 \\
\text { total sludge }^{\mathrm{c}} \\
\text { concentration) } \\
(\mu \mathrm{g} / \mathrm{g})\end{array}$ & $\begin{array}{c}241-\mathrm{S}-107 \\
\text { segments }^{\mathrm{c}} \\
(\mu \mathrm{g} / \mathrm{g})\end{array}$ & $\begin{array}{c}\text { Average } \\
\text { Concentration } \\
(\mu \mathrm{g} / \mathrm{g})\end{array}$ & $\begin{array}{l}\text { HDW sludge layer } \\
\text { concentration } \\
\text { for tank } \\
241-\mathrm{SX}-106^{\mathrm{e}} \\
(\mu \mathrm{g} / \mathrm{g})\end{array}$ \\
\hline $\mathrm{Al}$ & 127,000 & 117,000 & 56,400 & 100,000 & 24,700 \\
\hline $\mathrm{Bi}$ & $<38.8$ & $<45.7$ & NR & $<42.2$ & 1.06 \\
\hline $\mathrm{Ca}$ & 322 & 247 & 234 & 268 & 2,060 \\
\hline $\mathrm{Cl}$ & 2,050 & 3,200 & 1,860 & 2,370 & 2,770 \\
\hline $\mathrm{Cr}$ & 2,230 & 2,350 & 1,180 & 1,920 & 7,940 \\
\hline $\mathrm{F}$ & $<65.7$ & 145 & 150 & $<120$ & 5.5 \\
\hline $\mathrm{Fe}$ & 1,960 & 1,720 & 1,160 & 1,613 & 639 \\
\hline $\mathrm{Hg}$ & NR & $<0.126$ & NR & $<0.126$ & \\
\hline $\mathbf{K}$ & 539 & 300 & 457 & 432 & 667 \\
\hline $\mathrm{La}$ & $<19.5$ & $<2.07$ & NR & $<10.8$ & \\
\hline $\mathrm{Mn}$ & 2,750 & 1,150 & 83 & 1,330 & 2.02 \\
\hline $\mathrm{Na}$ & 112,000 & 121,000 & 60,400 & 97,800 & 137,000 \\
\hline $\mathrm{Ni}$ & 90.7 & 56 & 206 & 118 & 595. \\
\hline $\mathrm{NO}_{2}$ & 31,100 & 25,900 & 34,300 & 30,433 & 57,200 \\
\hline $\mathrm{NO}_{3}$ & 119,000 & 191,000 & 57,600 & 122,500 & 220,000 \\
\hline $\mathrm{Pb}$ & 37 & 29.6 & 33 & 33.2 & 26.1 \\
\hline $\mathrm{PO}_{4}$ & 1,360 & $<2,190$ & 1,630 & $<1,730$ & 31 \\
\hline $\mathrm{Si}$ & 1,360 & 1,330 & 1,060 & 1,250 & 2,420 \\
\hline $\mathrm{SO}_{4}$ & 897 & 2,270 & 1,300 & 1,489 & 2,850 \\
\hline $\mathrm{Sr}$ & 456 & 424 & 378 & 420 & $5.23 \mathrm{E}-06$ \\
\hline $\mathrm{TIC}$ as $\mathrm{CO}_{3}$ & NR & 4,140 & NR & 4,140 & \\
\hline TOC & NR & 1,730 & NR & 1,730 & 66 \\
\hline $\mathrm{U}$ & 7,684 & 6,690 & 8,685 & 7,690 & 5,410 \\
\hline $\mathrm{Zr}$ & 36 & 33.6 & 131 & 66.9 & 0.224 \\
\hline
\end{tabular}


HNF-SD-WM-ER-645

\section{Revision 0}

Table D3-4. R1 Sludge Concentration Estimate. (2 Sheets)

\begin{tabular}{|c|c|c|c|c|c|}
\hline Analyte & $\begin{array}{c}241-\mathrm{S}-101 \\
\text { segments } \\
7 \mathrm{U}-8 \mathrm{~L}^{\mathrm{a}} \\
(\mu \mathrm{g} / \mathrm{g})\end{array}$ & $\begin{array}{c}241-\mathrm{S}-104 \\
\text { total sludge }_{\text {concentration) }}^{\mathrm{b}} \\
(\mu \mathrm{g} / \mathrm{g})\end{array}$ & $\begin{array}{c}241-\mathrm{S}-107 \\
\text { segments } \\
(\mu \mathrm{g} / \mathrm{g})\end{array}$ & $\begin{array}{c}\text { Average } \\
\text { Concentration } \\
(\mu \mathrm{g} / \mathrm{g})\end{array}$ & $\begin{array}{c}\text { HDW sludge layer } \\
\text { concentration } \\
\text { for tank } \\
241-\mathrm{SX}-106^{\mathrm{e}} \\
(\mu \mathrm{g} / \mathrm{g})\end{array}$ \\
\hline \multicolumn{5}{|l|}{ Radionuclides $(\mu \mathrm{Ci} / \mathrm{g})$} \\
\hline${ }^{90} \mathrm{Sr}$ & $\mathrm{NR}$ & $301^{\mathrm{f}}$ & $276^{\mathrm{f}}$ & $288^{\mathrm{f}}$ & \\
\hline${ }^{137} \mathrm{Cs}$ & $98^{\mathrm{f}}$ & $60.5^{\mathrm{f}}$ & $74^{\mathrm{f}}$ & $77.6^{\mathrm{f}}$ & \\
\hline $\begin{array}{c}\text { density } \\
(\mathrm{g} / \mathrm{ml})\end{array}$ & 1.77 & 1.64 & 1.90 & 1.77 & 1.49 \\
\hline
\end{tabular}

HDW $=$ Hanford Defined Waste

$\mathrm{NR}=$ Not reported

REDOX $=$ Reduction oxidation process

R1 = REDOX waste generated between 1952 and 1957

${ }^{\text {a }}$ Kruger et al. (1996)

${ }^{b}$ DiCenso et al. (1994)

${ }^{c}$ Statistically determined median R1 sludge concentrations for tank 241-S-107 contained in the attachment to Simpson et al. (1996)

${ }^{d}$ Average of analyte concentrations for tank 241-S-101, 241-S-104, and 241-S-107

${ }^{e}$ Agnew et al. 1996

${ }^{\text {f }}$ Radionuclides decayed to January 1, 1994.

\section{D3.5 ESTIMATED COMPONENT INVENTORIES}

The Chemical inventory of tanks $241-\mathrm{SX}-106$ is estimated from the assumed salt cake and sludge volumes (Table D3-1). The resulting inventories are provided in Table D3-5. The inventories estimated by the HDW model are included for comparison.

Table D3-5. Comparison of Selected Component Inventory Estimates for Tank 241-SX-106. (2 Sheets)

\begin{tabular}{|c|c|c|c|c|}
\hline Component & $\begin{array}{c}\text { This evaluation } \\
(\mathrm{kg}) \text { sludge }\end{array}$ & $\begin{array}{c}\text { This evaluation } \\
(\mathrm{kg}) \text { salt cake }\end{array}$ & $\begin{array}{c}\text { This evaluation } \\
(\mathrm{kg})\end{array}$ & $\begin{array}{c}\text { HDW estimated } \\
(\mathrm{kg})\end{array}$ \\
\hline $\mathrm{Bi}$ & $<3.3$ & 457 & 462 & 578 \\
\hline $\mathrm{K}$ & 34 & 3,346 & 3,370 & 4,340 \\
\hline
\end{tabular}


Table D3-5. Comparison of Selected Component Inventory Estimates for Tank 241-SX-106. (2 Sheets)

\begin{tabular}{|c|c|c|c|c|}
\hline Component & $\begin{array}{c}\text { This evaluation } \\
(\mathrm{kg}) \text { sludge }\end{array}$ & $\begin{array}{c}\text { This evaluation } \\
(\mathrm{kg}) \text { salt cake }\end{array}$ & $\begin{array}{c}\text { This evaluation } \\
(\mathrm{kg})\end{array}$ & $\begin{array}{c}\text { HDW estimated } \\
(\mathrm{kg})\end{array}$ \\
\hline $\mathrm{La}$ & $<0.86$ & 20.7 & 21.6 & 12.7 \\
\hline $\mathrm{NO}_{3}$ & 9,760 & $1.07 \mathrm{E}+06$ & $1.08 \mathrm{E}+06$ & 648,000 \\
\hline $\mathrm{Mn}$ & 106 & 1,029 & 1,040 & 430 \\
\hline $\mathrm{SO}_{4}$ & 119 & 37,200 & 37,300 & 46,600 \\
\hline $\mathrm{Ni}$ & 9.4 & 254 & 263 & 761 \\
\hline $\mathrm{Cr}$ & 153 & 11,800 & 12,000 & 6,030 \\
\hline $\mathrm{PO}_{4}$ & $<137$ & 51,200 & 51,300 & 17,600 \\
\hline $\mathrm{F}$ & $<10$ & 4,600 & 4,610 & 2,960 \\
\hline $\mathrm{AI}$ & 7,916 & 36,500 & 44,500 & 83,600 \\
\hline $\mathrm{Fe}$ & 128 & 2,660 & 2,790 & 844 \\
\hline $\mathrm{TOC}$ & 138 & 13,200 & 13,300 & 25,300 \\
\hline $\mathrm{Na}$ & 7,790 & 605,000 & 613,000 & 532,000 \\
\hline $\mathrm{H}_{2} \mathrm{O}$ (percent) & $\mathrm{NR}$ & $\mathrm{NR}$ & 31.2 & 38.2 \\
\hline
\end{tabular}

HDW $=$ Hanford Defined Waste, Agnew et al. (1996)

$\mathrm{NR}=$ Not Recorded.

Since no post- 1989 analytical data were available for this tank, the reliability of these estimates (in either this engineering assessment or the HDW model inventory estimates) are suspect. Although these uncertainties cannot be resolved at this point, some trends can be discussed.

Manganese. Potassium permanganate was used in the REDOX process until 1959, thus manganese is expected to be found in tanks containing waste from that process. It is most likely present as highly insoluble manganese dioxide in the alkaline waste materials and would be expected to be in the sludge. The R1 Sludge composition estimate developed in this engineering assessment for Mn was $1,330 \mu \mathrm{g} / \mathrm{g}$. Interestingly, the SMMS1 salt cake composition estimate for $\mathrm{Mn}$ was $684 \mu \mathrm{g} / \mathrm{g}$--much higher than would be expected based on solubility considerations. It should be noted that there are large ranges in both the SMMS1 and $\mathrm{R} 1$ data sets for manganese.

The HDW model predicts approximately zero manganese in the sludge $(0.0114 \mathrm{~kg})$ in tank 241-SX-106 and $138 \mu \mathrm{g} / \mathrm{g}$ in the salt cake layer. The HDW model inventory estimate 
for manganese is $430 \mathrm{~kg}$. Based on the discussion above, the $1,140 \mathrm{~kg}$ inventory estimate developed in this engineering assessment is likely to be closer to the true value.

Phosphate. There is a large difference between the engineering assessment tank inventory estimate $(51,300 \mathrm{~kg}$ ) and the HDW model estimate $(17,600 \mathrm{~kg})$. The engineering assessment value is biased high because of one extremely high phosphate value in data set used to develop the SMMS1 salt cake composition estimate (see Table D3-2). If the phosphate data from tank 241-S-102 are eliminated from the SMMS1 composition estimate, then the engineering assessment and the HDW estimate would be in reasonable agreement. However, since the HDW model failed to predict the high phosphate value for tank 241-S-102, it should not be taken as a reliable indicator for phosphate in tank 241-SX-106.

Iron. The iron inventory estimate is about three times higher in the engineering assessment than in the HDW model. The iron value determined in the engineering assessment for the salt cake is approximately six times the HDW model value. As shown in Table D3-2, the data set used to estimate iron in the SMMS1 salt cake varies from $3,096 \mu \mathrm{g} / \mathrm{g}$ to less than detection limit and varies from 65 to $1,630 \mu \mathrm{g} / \mathrm{g}$ for the SMMS2 salt cake. Without analytical data from tank 241-SX-106 it is difficult to defend the choice of one value over the other.

Aluminum. The aluminum value determined in this engineering assessment is approximately half of the value predicted by the HDW model. As shown in Table D3-2, the aluminum values in the data from the four tanks are reasonably consistent. The reason for the two fold difference is unclear.

Nickel. The nickel inventory from the engineering assessment is approximately one third the value of the HDW model inventory. The HDW model predicts the majority of the nickel to be in the salt cake, as does the engineering assessment evaluation.

Total Hydroxide. Once the best-basis inventories were determined, the hydroxide inventory was calculated by performing a charge balance with the valence of other analytes. In some cases, this approach requires that other analyte (e.g., sodium or nitrate) inventories be adjusted to achieve the charge balance. During such adjustments, the number of significant figures is retained. This charge balance approach is consistent with that used by Agnew et al. (1997). 
HNF-SD-WM-ER-645

Revision 0

\section{D4.0 DEFINE THE BEST-BASIS AND ESTABLISH COMPONENT INVENTORIES}

Key waste management activities include overseeing tank farm operations and identifying, monitoring, and resolving safety issues associated with these operations and with the tank wastes. Disposal activities involve designing equipment, processes, and facilities for retrieving wastes and processing them into a form that is suitable for long-term storage/disposal. Information about chemical, radiological, and/or physical properties is used to perform safety analyses, engineering evaluations, and risk assessment associated with these activities.

Chemical and radiological inventory information are generally derived using three approaches: (1) component inventories are estimated using the results of sample analyses, (2) component inventories are predicted using the HDW model, process knowledge, and historical information, or (3) a tank-specific process estimate is made based on process flowsheets, reactor fuel data, essential material usage, and other operating data.

As part of this effort, an evaluation of available chemical information for tank 241-SX-106 was performed, including the following:

- The inventory estimate generated by the HDW model (Agnew et al. 1996).

- An engineering evaluation which produced a predicted SMMS salt cake and R1 inventory based on methodology developed by evaluation of similar $S$ and $U$ tanks and $\mathrm{R} 1$ inventory.

Based on this evaluation, a best-basis inventory was developed for tank 241-SX-106 since sampling information is not available. The engineering evaluation inventory was chosen as the best basis for those analytes for which sample-based analytical values were available, from $\mathrm{S}$ and U Tank Farm tanks with similar waste, for the following reasons:

- The sample-based inventory concentrations of the $S$ and $U$ Tank Farm tanks compared favorably with each other for SMMS1 salt cake, SMMS2 salt cake and R1 sludge.

- No methodology is available to fully predict SMMS1 and SMMS2 salt cake from process flowsheet or historical records.

- No methodology is available to fully predict $\mathrm{R} 1$ waste from process flowsheet or historical records for this tank. The REDOX process first-cycle R1 waste changed composition during the process and accurate records of these changes are not available at this time. Also R1 waste was cascaded and transferred into and out of many S, SX, and U Tank Farm tanks between 1972 and 1978 which makes it hard to predict precipitation factors for analytes in the waste. Some tanks will 
show higher concentrations for certain analytes because of the length of time the waste was in the tank before being transferred out.

- For those few analytes where no values were available from the sample-based inventory of similar tanks, the HDW model values were.

Best-basis tank inventory values are derived for 46 key radionuclides (as defined in Section 3.1 of Kupfer et al. 1997), all decayed to a common report date of January 1, 1994. Often, waste sample analyses have only reported ${ }^{90} \mathrm{Sr},{ }^{137} \mathrm{Cs},{ }^{239 / 240} \mathrm{Pu}$, and total uranium, or (total beta and total alpha) while other key radionuclides such as ${ }^{60} \mathrm{Co},{ }^{99} \mathrm{Tc},{ }^{129} \mathrm{I},{ }^{154} \mathrm{Eu},{ }^{155} \mathrm{Eu}$, and ${ }^{241} \mathrm{Am}$, etc., have been infrequently reported. For this reason it has been necessary to derive most of the 46 key radionuclides by computer models. These models estimate radionuclide activity in batches of reactor fuel, account for the split of radionuclides to various separations plant waste streams, and track their movement with tank waste transactions. (These computer models are described in Kupfer et al. 1997, Section 6.1 and in Watrous and Wootan 1997.) Model generated values for radionuclides in any of 177 tanks are reported in the HDW Rev. 4 model results (Agnew et al. 1997). The best-basis value for any one analyte may be either a model result or a sample or engineering assessment-based result if available. (No attempt has been made to ratio or normalize model results for all 46 radionuclides when values for measured radionuclides disagree with the model.) For a discussion of typical error between model derived values and sample derived values, see Kupfer et al. 1997, Section 6.1.10.

Best-basis tables for chemicals and only four radionuclides $\left({ }^{90} \mathrm{Sr},{ }^{137} \mathrm{Cs}, \mathrm{Pu}\right.$, and $\mathrm{U}$ ) were being generated in 1996, using values derived from an earlier version (Rev. 3) of the HDW model. When values for all 46 radionuclides became available in Rev 4 of the HDW model, they were merged with draft best-basis chemical inventory documents. Defined scope of work in FY 1997 did not permit Rev. 3 chemical values to be updated to Rev. 4 chemical values.

The best-basis inventory for tank 241-SX-106 is presented in Tables D4-1 and D4-2. The inventory values reported in Tables D4-1 and D4-2 are subject to change. Refer to the Tank Characterization Database (TCD) for the most current inventory values. 
HNF-SD-WM-ER-645

Revision 0

Table D4-1. Best-Basis Inventory Estimates for Nonradioactive Components in Tank 241-SX-106 (Effective January 31, 1997).

\begin{tabular}{|c|c|c|c|}
\hline Analyte & $\begin{array}{l}\text { Total inventory } \\
(\mathrm{kg})\end{array}$ & $\begin{array}{c}\text { Basis } \\
(\mathrm{S}, \mathrm{M}, \mathrm{C}, \text { or } \mathrm{E})^{1}\end{array}$ & Comment \\
\hline $\mathrm{Al}$ & 44,500 & $\mathrm{E}$ & \\
\hline $\mathrm{Bi}$ & 462 & E & \\
\hline $\mathrm{Ca}$ & 896 & $\mathrm{E}$ & \\
\hline $\mathrm{Cl}$ & 11,700 & $\mathrm{E}$ & \\
\hline $\mathrm{TIC}$ as $\mathrm{CO}_{3}$ & 52,100 & M & \\
\hline $\mathrm{Cr}$ & 12,000 & $\mathrm{E}$ & \\
\hline $\bar{F}$ & 4,610 & $\mathrm{E}$ & \\
\hline $\mathrm{Fe}$ & 2,790 & $E$ & \\
\hline $\mathrm{Hg}$ & 3.64 & $\mathrm{M}$ & \\
\hline $\mathrm{K}$ & 3,370 & $\mathrm{E}$ & \\
\hline $\mathrm{La}$ & 21.6 & $\mathrm{E}$ & \\
\hline $\mathrm{Mn}$ & 1,140 & $\mathrm{E}$ & \\
\hline $\mathrm{Na}$ & 613,000 & $\mathrm{E}$ & \\
\hline $\mathrm{Ni}$ & 263 & $\mathrm{E}$ & \\
\hline $\mathrm{NO}_{2}$ & 133,000 & $\mathrm{E}$ & \\
\hline $\mathrm{NO}_{3}$ & $1.08 \mathrm{E}+06$ & $\mathrm{E}$ & \\
\hline $\mathrm{OH}$ & 123,000 & $\mathrm{C}$ & \\
\hline $\mathrm{Pb}$ & 330 & $E$ & \\
\hline $\mathrm{PO}_{4}$ & 51,300 & $E$ & \\
\hline $\mathrm{Si}$ & 2,290 & $\mathrm{E}$ & \\
\hline $\mathrm{SO}_{4}$ & 37,300 & $E$ & \\
\hline $\mathrm{Sr}$ & 111 & $\mathrm{E}$ & \\
\hline TOC & 13,300 & $\mathrm{E}$ & \\
\hline $\mathrm{U}_{\text {TOTAL }}$ & 3,600 & $E$ & \\
\hline $\mathrm{Zr}$ & 63.2 & $\mathrm{E}$ & \\
\hline \multicolumn{4}{|c|}{$\begin{array}{l}{ }^{1} \mathrm{~S}=\text { Sample-based } \\
\mathrm{M}=\text { Hanford Defined Waste model-based (Agnew et al. 1996) } \\
\mathrm{E}=\text { Engineering assessment-based } \\
\mathrm{C}=\text { Calculated by charge balance; includes oxides as hydroxides, not including } \\
\mathrm{NO}_{2}, \mathrm{NO}_{3}, \mathrm{PO}_{4}, \mathrm{SO}_{4} \text {, and } \mathrm{SiO}_{3} .\end{array}$} \\
\hline
\end{tabular}




\section{Revision 0}

Table D4-2. Best-Basis Inventory Estimates for Radioactive Components in Tank 241-SX-106 (Decayed to January 1, 1994) (Effective January 31, 1997). (2 Sheets)

\begin{tabular}{|c|c|c|l|}
\hline Analyte & $\begin{array}{c}\text { Total inventory } \\
\text { (Ci) }\end{array}$ & $\begin{array}{c}\text { Basis } \\
\left(\mathrm{S}, \mathrm{M}, \text { or E) }{ }^{1}\right.\end{array}$ & Comment \\
\hline${ }^{3} \mathrm{H}$ & 513 & $\mathrm{M}$ & \\
\hline${ }^{14} \mathrm{C}$ & 73.7 & $\mathrm{M}$ & \\
\hline${ }^{59} \mathrm{Ni}$ & 4.8 & $\mathrm{M}$ & \\
\hline${ }^{60} \mathrm{Co}$ & 81.6 & $\mathrm{M}$ & \\
\hline${ }^{63} \mathrm{Ni}$ & 470 & $\mathrm{M}$ & \\
\hline${ }^{79} \mathrm{Se}$ & 7.35 & $\mathrm{M}$ & \\
\hline${ }^{90} \mathrm{Sr}$ & 309,000 & $\mathrm{E}$ & \\
\hline${ }^{90} \mathrm{Y}$ & 309,000 & $\mathrm{E}$ & Referenced to ${ }^{90} \mathrm{Sr}$ \\
\hline${ }^{93} \mathrm{Zr}$ & 36.1 & $\mathrm{M}$ & \\
\hline${ }^{93 \mathrm{~m}} \mathrm{Nb}$ & 26.2 & $\mathrm{M}$ & \\
\hline${ }^{99} \mathrm{Tc}$ & 525 & $\mathrm{M}$ & \\
\hline${ }^{106} \mathrm{Ru}$ & 0.0145 & $\mathrm{M}$ & \\
\hline${ }^{113 \mathrm{~m}} \mathrm{Cd}$ & 189 & $\mathrm{M}$ & \\
\hline${ }^{125} \mathrm{Sb}$ & 351 & $\mathrm{M}$ & \\
\hline${ }^{126} \mathrm{Sn}$ & 11.1 & $\mathrm{M}$ & \\
\hline${ }^{129} \mathrm{I}$ & 1.01 & $\mathrm{M}$ & \\
\hline${ }^{134} \mathrm{Cs}$ & 5.57 & $\mathrm{M}$ & \\
\hline${ }^{137} \mathrm{Cs}$ & 398,000 & $\mathrm{E}$ & \\
\hline${ }^{137 m} \mathrm{Ba}$ & 376,000 & $\mathrm{E}$ & Referenced to ${ }^{137} \mathrm{Cs}$ \\
\hline${ }^{151} \mathrm{Sm}$ & 25,900 & $\mathrm{M}$ & \\
\hline${ }^{152} \mathrm{Eu}$ & 8.49 & $\mathrm{M}$ & \\
\hline${ }^{154} \mathrm{Eu}$ & 1,330 & $\mathrm{M}$ & \\
\hline${ }^{155} \mathrm{Eu}$ & 501 & $\mathrm{M}$ & \\
\hline${ }^{226} \mathrm{Ra}$ & $3.15 \mathrm{E}-04$ & $\mathrm{M}$ & \\
\hline${ }^{227} \mathrm{Ac}$ & 0.00199 & $\mathrm{M}$ & \\
\hline${ }^{228} \mathrm{Ra}$ & 0.307 & $\mathrm{M}$ & \\
\hline${ }^{229} \mathrm{Th}$ & 0.0072 & $\mathrm{M}$ & \\
\hline${ }^{231} \mathrm{~Pa}$ & 0.00913 & $\mathrm{M}$ & \\
\hline & & & \\
\hline
\end{tabular}




\section{Revision 0}

Table D4-2. Best-Basis Inventory Estimates for Radioactive Components in Tank 241-SX-106 (Decayed to January 1, 1994) (Effective January 31, 1997). (2 Sheets)

\begin{tabular}{|c|c|c|l|}
\hline Analyte & $\begin{array}{c}\text { Total inventory } \\
\text { (Ci) }\end{array}$ & $\begin{array}{c}\text { Basis } \\
(\mathrm{S}, \mathrm{M}, \text { or } \mathrm{E})^{1}\end{array}$ & Comment \\
\hline${ }^{232} \mathrm{Th}$ & 0.0204 & $\mathrm{M}$ & \\
\hline${ }^{232} \mathrm{U}$ & 1.58 & $\mathrm{M}$ & \\
\hline${ }^{233} \mathrm{U}$ & 6.05 & $\mathrm{M}$ & \\
\hline${ }^{234} \mathrm{U}$ & 1.77 & $\mathrm{M}$ & \\
\hline${ }^{235} \mathrm{U}$ & 0.0719 & $\mathrm{M}$ & \\
\hline${ }^{236} \mathrm{U}$ & 0.0555 & $\mathrm{M}$ & \\
\hline${ }^{237} \mathrm{~Np}$ & 1.92 & $\mathrm{M}$ & \\
\hline${ }^{238} \mathrm{Pu}$ & 3.0 & $\mathrm{M}$ & \\
\hline${ }^{238} \mathrm{U}$ & 2.05 & $\mathrm{M}$ & \\
\hline${ }^{239} \mathrm{Pu}$ & 103 & $\mathrm{M}$ & \\
\hline${ }^{240} \mathrm{Pu}$ & 17.5 & $\mathrm{M}$ & \\
\hline${ }^{241} \mathrm{Am}$ & 125 & $\mathrm{M}$ & \\
\hline${ }^{241} \mathrm{Pu}$ & 203 & $\mathrm{M}$ & \\
\hline${ }^{242} \mathrm{Cm}$ & 0.32 & $\mathrm{M}$ & \\
\hline${ }^{242} \mathrm{Pu}$ & 0.00111 & $\mathrm{M}$ & \\
\hline${ }^{243} \mathrm{Am}$ & 0.00434 & $\mathrm{M}$ & \\
\hline${ }^{243} \mathrm{Cm}$ & 0.0297 & $\mathrm{M}$ & \\
\hline${ }^{244} \mathrm{Cm}$ & 0.293 & $\mathrm{M}$ & \\
\hline & & & \\
\hline
\end{tabular}

${ }^{1} \mathrm{~S}=$ Sample-based

$\mathrm{M}=$ Hanford Defined Waste model-based (Agnew et al. 1997)

$\mathrm{E}=$ Engineering assessment-based. 
HNF-SD-WM-ER-645

Revision 0

This page intentionally left blank.

D-20 
HNF-SD-WM-ER-645

Revision 0

\section{D5.0 APPENDIX D REFERENCES}

Agnew, S. F., J. Boyer, R. A. Corbin, T. B. Duran, J. R. FitzPatrick, K. A. Jurgensen, T. P. Ortiz, and B. L. Young, 1996, Hanford Tank Chemical and Radionuclide Inventories: HDW Model Rev. 3, LA-UR-96-858, Los Alamos National Laboratory, Los Alamos, New Mexico.

Agnew, S. F., J. Boyer, R. A. Corbin, T. B. Duran, J. R. FitzPatrick, K. A. Jurgensen, T. P. Ortiz, and B. L. Young, 1997, Hanford Tank Chemical and Radionuclide Inventories: HDW Model Rev. 4, LA-UR-96-3860, Rev. 4, Los Alamos National Laboratory, Los Alamos, New Mexico.

Baldwin, J. H., and R. H. Stephens, 1996, Tank Characterization Report for Single-Shell Tank 241-U-109, WHC-SD-WM-ER-609, Rev. 0, Westinghouse Hanford Company, Richland, Washington.

Brown, T. M., R. D. Cromar, J. L. Strope, and R. T. Winward, 1997, Tank Characterization Report for Single-Shell Tank 241-U-106, HNF-SD-WM-ER-636, Rev. 0, Lockheed Martin Hanford Corporation, Richland, Washington.

DiCenso, A. T., L. C. Amato, J. D. Franklin, G. L. Nuttall, K. W. Johnson, P. Sathyanarayana, and B. C. Simpson, 1994, Tank Characterization Report for SingleShell Tank 241-S-104, WHC-SD-WM-ER-370, Rev. 0, Westinghouse Hanford Company, Richland, Washington.

Eggers, R. F., R. H. Stephens, and T. T. Tran, 1996, Tank Characterization Report for Single-Shell Tank 241-S-102, WHC-SD-WM-ER-611, Rev. 0, Westinghouse Hanford Company, Richland, Washington.

Hanlon, B. M., 1996, Waste Tank Summary Report for Month Ending June 30, 1996, WHC-EP-0182-99, Westinghouse Hanford Company, Richland, Washington.

Hill, J. G., G. S. Anderson, and B. C. Simpson, 1995, The Sort on Radioactive Waste Type Model: A Method to Sort Single-shell Tanks into Characteristic Groups, PNL-9814, Rev. 2, Pacific Northwest Laboratory, Richland, Washington.

Hodgson, K. M., and M. D. LeClair, 1996, Work Plan for Defining a Standard Inventory Estimate for Wastes Stored in Hanford Site Underground Tanks, WHC-SD-WM-WP-311, Rev. 1, Lockheed Martin Hanford Corporation, Richland, Washington.

Hu, T.A., L. C. Amato, R. T. Winward, S. R. Wilmarth, and J. L. Stroup, 1997, Tank Characterization Report for Single-Shell Tank 241-U-102, HNF-SD-WM-ER-618, Rev. 0, Lockheed Martin Hanford Corporation, Richland, Washington. 
Jo, J., B. J. Morris, and T. T. Tran, 1996, Tank Characterization Report for Single-Shell Tank 24I-U-107, WHC-SD-WM-ER-614, Rev. 0, Westinghouse Hanford Company, Richland, Washington.

Kruger, A. A., B. J. Morris, and L. J. Fergestrom, 1996, Tank Characterization Report for Single-Shell Tank 241-S-101, WHC-SD-WM-ER-613, Rev. 0, Westinghouse Hanford Company, Richland, Washington.

Kupfer, M. J., A. L. Boldt, B. A. Higley, K. M. Hodgson, L. W. Shelton, B. C. Simpson, and R. A. Watrous (LMHC), S. L. Lambert, and D. E. Place (SESC), R. M. Orme (NHC), G. L. Borsheim (Borsheim Associates), N. G. Colton (PNNL), M. D. LeClair (SAIC), R. T. Winward (Meier Associates), and W. W. Schulz (W'S Corporation), 1997, Standard Inventories of Chemicals and Radionuclides in Hanford Site Tank Wastes, HNF-SD-WM-TI-740, Rev. 0, Lockheed Martin Hanford Corporation, Richland, Washington.

Simpson, B. C., J. G. Field, D. W. Engel, and D. S. Daly, 1996, Tank Characterization Report for Single-Shell Tank 241-S-107, WHC-SD-WM-ER-589, Rev. 0, Westinghouse Hanford Company, Richland, Washington.

Watrous, R. A., and D. W. Wootan, 1997, Activity of Fuel Batches Processed Through Hanford Separations Plants, 1944 Through 1989, HNF-SD-WM-TI-794, Rev. 0, Lockheed Martin Hanford Corporation, Richland, Washington. 